\title{
Construction of a tape-controlled system of presenting visual-spatial and visual-temporal stimulus patterns for use in cross-modal and intramodal matching tests
}

\author{
RONALD F. JARMAN, MALCOLM F. MARSHALL, and TOM S. MOORE \\ University of British Columbia, Vancouver, British Columbia V6T IW5, Canada
}

\begin{abstract}
A technique of tape construction is described for use in the generation and control of all combinations of auditory-temporal, visual-temporal, and visual-spatial stimulus patterns. This technique allows the development of a set of nine tasks for testing cross-modal and intramodal matching.
\end{abstract}

Sensory modality matching tasks, as used in a substantial number of research studies, have presented several notable problems and limitations. Prominent among these is the confounding of auditory-visual and temporal-spatial elements of stimulus presentation (Rubinstein \& Gruenberg, 1971; Rudnick, Martin, \& Sterritt, 1972; Sterritt, Martin, \& Rudnick, 1971), such that possible sensory system differences have been indistinguishable from the temporal-spatial dimensions. In addition, manually controlled methods of stimulus presentation have added variability due to experimenter judgment and reaction time differences.

Jarman (1977) discussed techniques of constructing auditory-temporal stimulus patterns for modality matching tests and presented a new method for constructing such stimulus patterns. As a further development of that technique, a method is now presented to include visual-spatial and visual-temporal test conditions in a complete cassette tape-controlled series of modality matching tests, which corrects the inadequacies previously mentioned.

\section{TEST DESCRIPTIONS}

The tests are comprised of three conditions: auditory. temporal (AT), visual-spatial (VS), and visual-temporal (VT) patterns, presented respectively by tape recorder, slides, and a flashing light. These conditions are presented in pairs to form matching tasks with an original or standard stimulus followed by a matching or comparison stimulus (Sterritt et al,, 1971). This permits nine combinations of matching, with each condition appearing in either or both standard and comparison positions when paired with each of the

Requests for reprints should be sent to Ronald $F$. Jarman, Department of Educational Psychology, Faculty of Education, University of British Columbia, Vancouver, British Columbia, Canada V6T $1 \mathrm{~W} 5$. other elements or with itself: AT-AT, AT-VT, AT-VS, VT-AT, VT-VT, VT-VS, VS-AT, VS-VT, and VS-VS. In the particular example described here, the AT condition (Jarman, 1977) consists of $1,000-\mathrm{Hz}$ tones, all $.15 \mathrm{sec}$ in duration, with short pauses of $.35 \mathrm{sec}$ and long pauses of $1.35 \mathrm{sec}$. The VS condition is made up of dots in a linear horizontal format, such that with dots of 1 unit in diameter, short gaps between dots are .80 units and long gaps are 7.17 units. The VT condition is composed of flashes of a $100-\mathrm{mA}$ lamp, which are identical in timing and duration to the AT patterns.

The four matching tests outlined by Jarman (1977), which were AT-AT, AT-VS, VS-AT, and VS-VS, remain the same, apart from the addition of programmed inaudible tone bursts (sync pulses) on the cue track of the cassette tape for advancing the projector in the VS condition of the tests. These pulses permit presentation of the slides for a time proportional to the total duration of temporal patterns, which ranges from 2 to $4 \mathrm{sec}$ in this particular set of items. The verbal instructions and $1,000-\mathrm{Hz}$ tones are recorded on one track of a variable speed, reel-to-reel, two-track tape recorder at $7-1 / 2$ ips. Pulses of $250 \mathrm{~Hz}$ are recorded on the second track, running at $1-7 / 8$ ips. When the tape is played at the original speed of $7-1 / 2$ ips, the cue track transfers at $1,000 \mathrm{~Hz}$, with a slide duration error of less than $\pm .2 \mathrm{sec}$.

\section{CONSTRUCTION OF VISUALTEMPORAL CONDITION}

For construction of the VT stimulus patterns, a Wollensak 3M 2551 AV slide/sync cassette tape recorder was used. The VT condition requires that a small incandescent lamp be flashed by a pattern of inaudible tones, but these tones cannot be directly transferred to the cue track, since this track already contains $1,000-\mathrm{Hz}$ tones as slide-change cues. This problem is overcome by building two electronic circuits. 


\section{Tone Decoder}

The tone decoder circuit is comprised of a differential amplifier (National LM741), a tone decoder, and a discrete lamp driver. This is connected directly to the cue track record-playback tape head. Signals from the head are amplified and sent to the tone decoder, which is programmed to respond to $500-\mathrm{Hz}$ tones $( \pm 10 \mathrm{~Hz}$ for tape speed variation). The response of the decoder signals the lamp driver to turn on a $12-\mathrm{V} 100-\mathrm{mA}$ incandescent lamp for the duration of the $500-\mathrm{Hz}$ tone bursts.

The decoder is coupled to the tape recorder by a two-conductor shielded cable, and the "sync out" of the recorder is connected to the "sync in" of the decoder. An extension cord to the lamp is plugged into a socket on the top of the decoder. Power to the decoder is controlled by a switch on the decoder box, and power is indicated by the light-emitting diode (LED). A pushbutton switch on top of the decoder permits testing and manual control of the lamp.

\section{Binary Divider}

The original audible $1,000-\mathrm{Hz}$ tone bursts are played back through the binary circuit and divided to $500 \mathrm{~Hz}$. These $500-\mathrm{Hz}$ tone bursts are simultaneously rerecorded onto the inaudible cue track. The slide changer thus responds only to the $1,000-\mathrm{Hz}$ tones.

The speaker output from the recorder is connected via a $\mathrm{Y}$ cord to an auxiliary speaker and to the divider input of the decoder. The volume control is set so that approximately $4.5 \mathrm{~V}$ peak to peak (p-p) is fed to the divider input. This is the value required for the divider to operate properly.

For tones recorded on the Wollensak $2551 \mathrm{AV}$ recorder at $0 \mathrm{VU}$ (100\% modulation), an oscilloscope and/or frequency counter can be placed on the divider output and the volume control turned up until a stable square-wave output of $500 \mathrm{~Hz}$ shows on the oscilloscope. An audible distortion will be evident on the auxiliary speaker.

The output of the divider is fed into the sync input of the recorder via a shielded single-conductor cable. The output gain can be controlled via the out gain on the decoder. This should be set for $1 \mathrm{~V}$ nominal. Too large a signal causes the projector to advance; a weak signal will not light the lamp properly. Divider and decoder sections should not be hooked up at the same time, and power should not be turned on until all connections are made. The lamp should always be connected to the decoder, whether operating the decoder or divider.

\section{CIRCUIT DESCRIPTIONS}

\section{Power Supply}

The power supply provides $\pm 15 \mathrm{~V}$ and $+5 \mathrm{~V}$. The line level is transformed and rectified (D1-D4; see Figure 1) and filtered $(\mathrm{C} 17, \mathrm{C} 18)$. This voltage, $\pm 35 \mathrm{~V}$, is then regulated to $+15 \mathrm{~V}$ by IC 6 and to $-15 \mathrm{~V}$ by IC7. A +5-V source is obtained by IC 5 .

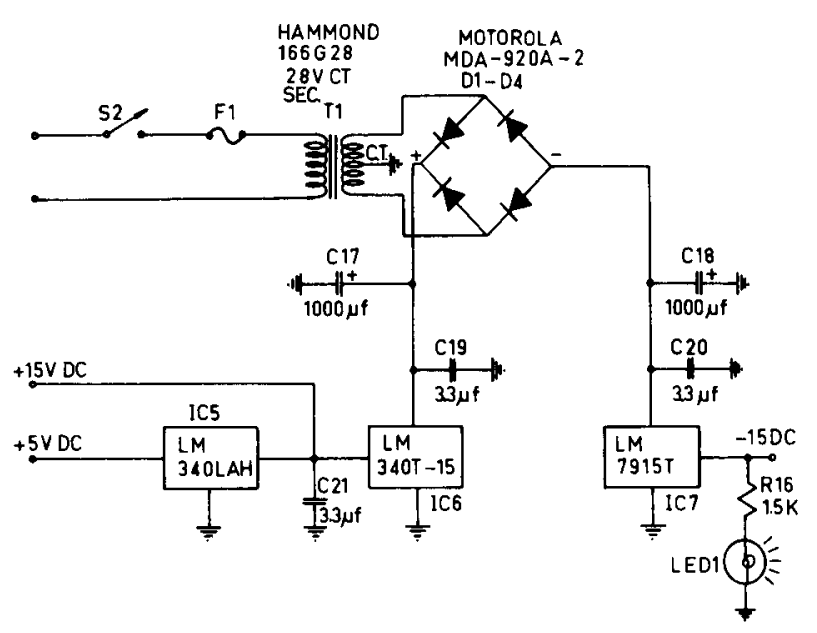

Figure 1. Power supply.

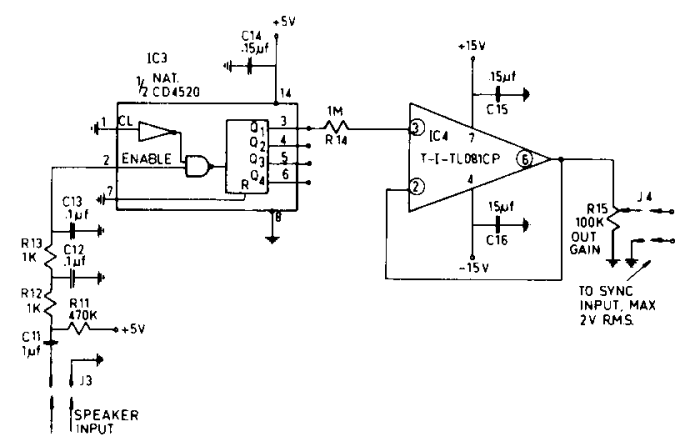

Figure 2. Divide by two divider and driver.

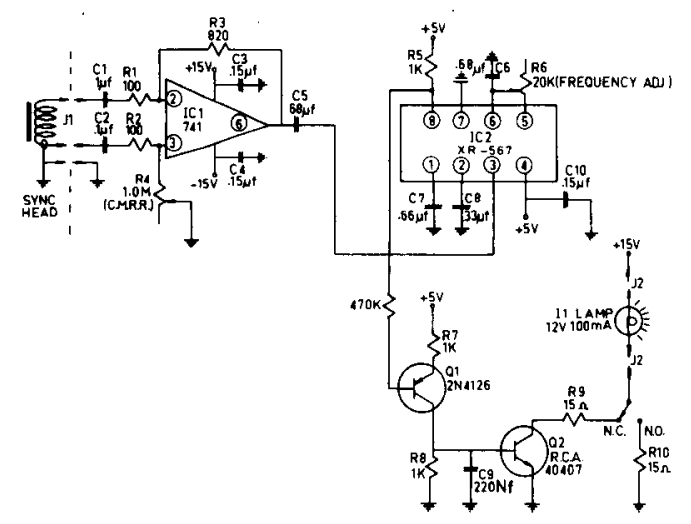

Figure 3. The 500-Hz decoder and lamp driver.

\section{Divider Section}

The signal from the tape-recorder speaker is fed through $\mathrm{C} 11$ (see Figure 2) to a low-pass filter (C12, $\mathrm{C} 13, \mathrm{R} 12, \mathrm{R} 13)$ to eliminate any high-frequency component from affecting the divider. The signal is then fed to half of IC3, a dual binary divider. The divider output is fed to a unity gain Op Amp IC4, and the output, controlled by R15, is fed back to the taperecorder sync input.

\section{Tone Decoder}

The signal from the sync head is coupled via $\mathrm{C} 1, \mathrm{C} 2$ 
to a differential amplifier IC1 (see Figure 3). The common mode rejection ratio (R4) is adjusted for minimum hum at $60 \mathrm{~Hz}$. The output of IC1 is coupled to a tone decoder IC2. This decoder's free running frequency is adjusted by $\mathrm{C} 6$ and $\mathrm{R} 6$ (set to $500 \mathrm{~Hz}$ ); the decoding section is set by the two loop filters $\mathrm{C} 7$ and C8. The output of the decoder is inverted by Q1 and fed to Q2, which acts as an inverter and lamp driver. The filter C9 prevents superfluous oscillations. A switch is also included for manual operation of the lamp. Construction of the five new tapes for the addition of the visual-temporal element is achieved by dividing the $1,000-\mathrm{Hz}$ audible sound patterns on copies of the three original tapes that contain the auditory-temporal condition and simultaneously rerecording them on the cue track. Once the transfer is made, the original audible tone bursts are erased from the audio track. The AT-AT test becomes VT-VT, and the AT-VS test and its converse become the VT-VS test and its converse. When the AT-AT test has the initial stimuli altered, it becomes VT-AT; when the comparison half is altered, this becomes AT.VT.

Through use of the procedures in Jarman (1977), followed by those described here, a complete set of cross-modal and intramodal tasks may be constructed to any specifications. If additional precision is desired in the timing of the visual-temporal condition, a strobe light may be substituted for the incandescent lamp, to avoid the slower rise and fall of light and slight instability due to temperature in incandescent sources. Also, this light may be filtered to narrow the frequency band from white light, making it more comparable to the single frequency of the tones in the auditory-temporal condition.

\section{REFERENCES}

JARMAN, R. F. A method of construction of auditory stimulus patterns for use in cross-modal and intramodal matching tests. Behavior Research Methods \& Instrumentation, 1977, 9, 22-25.

Rubinstein, L., \& Gruenberg, E. M. Intramodal and crossmodal sensory transfer and auditory temporal patterns. Perception \& Psychophysics, 1971, 9, 385-390.

Rudnick, M., Martin, V., \& Sterritt, G. M. On the relative difficulty of auditory and visual, temporal and spatial, integrative and nonintegrative sequential pattern comparisons. Psychonomic Science, 1972, 27, 207-210.

Sterritt, G. M., Martin, V., \& Rudnick, M. Auditoryvisual and temporal-spatial integration as determinants of test difficulty. Psychonomic Science, 1971, 23, 289-291.

(Received for publication June 29, 1978; revision accepted December 27, 1978.) 